\title{
Farmers Suicides in Marathwada Region of India: A Causative Analysis
}

\author{
B. Venkateswarlu', R.D. Ahire ${ }^{2}$ and P.S. Kapse ${ }^{2 *}$ \\ ${ }^{1}$ VNMKV, Parbhani, \\ ${ }^{2}$ Department of Extension Education, VNMKV, Parbhani, \\ Vasantrao Naik Marathwada Krishi Vidyapeeth, Parbhani - 431 402, Maharashtra, India \\ *Corresponding author
}

\section{A B S T R A C T}

The present investigation was undertaken in eight districts of the Marathwada region of Maharashtra State (India) to study the socio-economic, psychological and situational causes of farmers' suicide. Data was collected from the family members of the victims

\section{Keywords}

Farmers suicide, Socio-economic causes, Marathwada region of India

Article Info

Accepted: 04 March 2019 Available Online: 10 April 2019 with the help of a specially designed interview schedule. Statistical tools like mean, standard deviation, percentage and frequency were used. Results revealed that majority of the victims were in middle age, educated up to high school, annual income between Rs. $50001 /$ - to 150000 /-, and marginal to small land holders. Only 4.06 per cent of the victims had subsidiary occupation. Majority of the victims are rainfed farmers with no access to irrigation. Majority of victims had substantial outstanding of the loans from banks and money lenders. Crop failure due to drought / lack of access to irrigation was the primary cause, followed by high indebtedness. Drop in socio-economic status, addiction and huge expenses on marriages of daughter were other social causes. Remedial measures received from family members included remunerative prices for farm produce, simplification of procedure for crop insurance and timely crop loan at minimum interest. Support for digging wells, subsidised seed and fertilizer supply to poor farmers, technical back stopping and subsidy to take up subsidiary occupation were other major suggestions received from family members of the victims.

\section{Introduction}

Farmer suicides have become a major concern in India with profound socio-economic implications. The United Nations Commission on Sustainable Development (UNCSD) reported that in India about one farmer ended his life every 32 minutes between 1997 and 2005. According to National Crime Records Bureau (NCRB), the tendency of states like Maharashtra, Telangana, Madhya Pradesh, Chhattisgarh and Karnataka have recorded alarming rates of farmer suicides during 2014. These five states together account for 90 per cent of farmer suicides (5056) in India. This was further increased by 42 per cent in 2015 . Fifteen farmers committed suicide every day in the country during 2014 and which went up to 21 in 2015. 
Farmers' suicides in Maharashtra and Marathwada

Maharashtra recorded 51837 farmer suicides during 1995-2014, which represents 22.50 per cent of total suicides in the states. The number of farmers' suicide has increased sharply in Maharashtra than other states. Considering the base year 1995, farmers' suicides have increased by 284 per cent during the first decade (1995-2004) and 270 per cent during second decade (2005-2014). Data also show that majority of the farmer suicides were in the Vidharbha and Marathwada.

Data on the total farmers' suicides in the Marathwada along with district wise breakup is presented in Table 1. It is observed that 1133, 1053 and 991 farmers committed suicides in the year 2015, 2016 and 2017 respectively from the region. The present investigation therefore was undertaken in the eight districts of the Marathwada region with followings objectives;

To study the personal, socio-economical, psychological and situational characteristics of the victims family who commit suicides.

To assess the socio-economic, psychological and situational causes which compelled the victims to commit suicide.

\section{Materials and Methods}

The study was conducted in all eight districts of the Marathwada region viz., Parbhani (PBN), Hingoli (HGL), Nanded (NND), Beed, Jalna (JLN), Osmanabad (O’BAD), Latur (LTR) and Aurangabad (A'BAD). From these eight districts, 320 households were selected in which a farmer committed suicide during last three years (i.e. 2014, 2015 and 2016). The data was collected from the family members of victims, relatives, neighbours and key informants etc. of the village by personally interviewing them with the help of a specially designed interview schedule. Secondary data on number of farmers' suicides was collected from National Crime Records Bureau (NCRB). Statistical tools like mean, standard deviation, percentage and frequency were used.

\section{Results and Discussion}

Personal, socio-economical, psychological and situational characteristics of the victims families who committed suicides

It is observed from Table 2 that 39.68 per cent of the victims were middle age (36 to 50 years), while 31.56 per cent of them were young age (upto 35 years), followed by 28.76 per cent were old age group. It means that most of victims were below the age of 50 years.

Table 3 indicated that 21.87 per cent victim were educated upto high school level, followed by 21.25 per cent having primary school level education; whereas 20.62 per cent were illiterate and 18.13 per cent were having the higher school. Whereas, 5.32 and 0.63 per cent were educated upto graduation and post graduate level education, respectively. Majority of the victims were educated upto middle school level.

Table 4 shows that the information of land holding of the victims, the data revealed that 39.68 and 31.57 per cent victims were having small (1.01 ha to 2.00) and marginal (upto 1.00 ha) land holding, respectively and 21.25 per cent victims was from semi-medium (2.01 to 4.00 ha.) type of land holding. Whereas, 6.56 and 00.94 per cent of victims were from medium (4.01 to $10.00 \mathrm{ha}$.) and large holdings (above 10.00 ha.), respectively. It shows that majority of the victims were marginal and small farmers. Subsidiary occupation is one of the important components for farming community in addition to agriculture 
enterprise. It is seen from Table 5 that more than half of the victims were having the occupation i.e. Agriculture + Labour (61.25\%), followed by 21.88 per cent were having sole farming as their main occupation, whereas 10.31 per cent, 4.06 per cent and 2.50 per cent victims were having Agriculture + Business, Agriculture + Allied occupation and Agriculture + Service as the occupation, respectively. It is inferred that very small per cent of victims were having allied occupations along with agriculture.

Table 6 data revealed that nearly half of the victims $(49.07 \%)$ were having annual income between Rs. 50,001 /- to Rs. 1,50,000 /- while 21.87 per cent of victim's income was between Rs. 27,001 /- to Rs. 50,000 /-, followed by 14.37 per cent, 7.50 per cent and 5.94 per cent of victims having income in between Rs. 1,50,001 /- to Rs. 2,50,000 /-, Rs.2,50,001 /- to Rs. 6,00,000/- and upto Rs 27,000 , respectively. Whereas 1.25 per cent of the victims were having the annual income of above Rs. 6,00,000/-. It means that more than three fourth of the victims were having annual income only upto Rs. 1,50,000/-.

Sources of irrigation data in Table 7 shows that 53.74 per cent of victims were not having any source of irrigation, followed by 31.56 per cent victims were having well and tube well as source of irrigation, while 10.00 per cent victims were having canal as source of irrigation.

It is observed from Table 8 that 82.18 per cent of the victims had adopted sole cropping system whereas only 17.82 per cent adopted intercropping. While as per Table 9, it is depicted that average productivity of major kharif crops viz., soybean, cotton, and pigeonpea of the victims were $9.56 \mathrm{qt} / \mathrm{ha}$, $11.20 \mathrm{qt} / \mathrm{ha}$ and $7.34 \mathrm{q} / \mathrm{ha}$, respectively. Among rabi crops, productivity of bengal gram, rabi sorghum and wheat were 7.20 qt/ha, $9.65 \mathrm{qt} / \mathrm{ha}$ and $11.99 \mathrm{qt} / \mathrm{ha}$, respectively.

With regard to credit and indebtedness of the victims, it is observed (Table 10) that 54.06 per cent of victims had taken the loan from nationalised banks, followed by 26.56 per cent from Co-operative banks and 11.56 per cent from rural banks as institutional credit source. While 36.25 per cent of the victims had taken loans from private money lenders, followed by 28.43 per cent from friends and relatives as non-institutional credit source.

Livelihood pattern of victims are depicted in Table 11. Nearly all victims had agriculture as a sole source of income with an average annual income of Rs. 74,576 /- per victim, followed by 61.25 per cent of victims had wages as a source of income with an average annual income of Rs. 30988 /- per victim. Whereas only 4.06 per cent of them had allied occupation as a source of income with an average annual income of Rs. 21,176 /- per victim. It means that main source of income of victims was only from agriculture.

\section{Compelling socio-economic, psychological and situational causes of farmers suicides}

Table 12 shows various farm related causes driving victims for suicide. It is seen that cause for 87.18 per cent of victims was failure of crop due to drought / lack of irrigation, followed by 5.62, 3.12 and 2.81 per cent were crop failure due to hailstorm, failure of open well or tube wells and crop failure due to unseasonal rains. Few percentage of the victims cause for suicide was crop failure due to infestation of insect / disease $(0.93 \%)$ and due to poor seed germination $(0.62 \%)$. Indebtedness causes of farmers suicide is given in Table 13, it was indicated that 76.56 per cents cause for suicide was increased indebtedness, followed by recovery pressure from money lenders (25.62\%), torturing by money lender for repayment of loan (21.56 
$\%$ ), humiliation in public by money lender $(9.06 \%)$, availment of loan from noninstitutional sources $(7.18 \%)$ and auction of assets / livestock and other household stocks (6.87\%), respectively.

Social and other causes related to farmer suicides are presented in Table 14. Data revealed that 53.12 per cent of victims cause for suicide was drop in socio-economic status, followed by addiction of victims (40.00\%), chronic health problem of victims or family members $(33.75 \%)$, marriageable age of daughters / sisters $(30.31 \%)$ and suicide of family members or any peers $(20.31 \%)$. While dispute with the family member or neighbour or others was the cause for suicide of 9.69 per cent victims and death of the family member prior to suicide also the cause in 3.12 per cent cases. Only 1.87 per cent of the victims cause for suicide was dowry system.

Table.1 Number of farmers suicides in Marathwada region

\begin{tabular}{|c|c|c|c|c|c|c|c|c|c|}
\hline Year & Aurangabad & Latur & Beed & Parbhani & Jalna & Hingoli & Osmanabad & Nanded & Total \\
\hline $\mathbf{2 0 1 0}$ & 02 & 04 & 79 & 22 & 04 & 02 & 23 & 55 & $\mathbf{1 9 1}$ \\
\hline $\mathbf{2 0 1 1}$ & 00 & 04 & 73 & 23 & 06 & 05 & 25 & 33 & $\mathbf{1 6 9}$ \\
\hline $\mathbf{2 0 1 2}$ & 02 & 00 & 91 & 35 & 06 & 03 & 22 & 39 & $\mathbf{1 9 8}$ \\
\hline $\mathbf{2 0 1 3}$ & 04 & 03 & 98 & 17 & 08 & 02 & 29 & 46 & $\mathbf{2 0 7}$ \\
\hline $\mathbf{2 0 1 4}$ & 56 & 44 & 152 & 70 & 32 & 31 & 71 & 118 & $\mathbf{5 7 4}$ \\
\hline $\mathbf{2 0 1 5}$ & 144 & 106 & 301 & 104 & 83 & 41 & 164 & 190 & $\mathbf{1 1 3 3}$ \\
\hline $\mathbf{2 0 1 6}$ & 151 & 116 & 222 & 98 & 76 & 49 & 161 & 180 & $\mathbf{1 0 5 3}$ \\
\hline $\mathbf{2 0 1 7}$ & 139 & 94 & 207 & 125 & 91 & 56 & 126 & 153 & $\mathbf{9 9 1}$ \\
\hline Total & $\mathbf{4 9 8}$ & $\mathbf{3 7 1}$ & $\mathbf{1 2 2 3}$ & $\mathbf{4 9 4}$ & $\mathbf{3 0 6}$ & $\mathbf{1 8 9}$ & $\mathbf{6 2 1}$ & $\mathbf{8 1 4}$ & $\mathbf{4 5 1 6}$ \\
\hline
\end{tabular}

Table.2 Distribution of the victims according to their age

\begin{tabular}{|c|l|c|c|c|}
\hline Sr. No & \multicolumn{1}{|c|}{ Districts } & \multicolumn{3}{|c|}{ Age } \\
\cline { 3 - 5 } & & $\begin{array}{c}\text { Young } \\
\text { (Up to 35) }\end{array}$ & $\begin{array}{c}\text { Middle } \\
\text { (36 to 50) }\end{array}$ & $\begin{array}{c}\text { Old } \\
\text { (Above 51) }\end{array}$ \\
\hline $\mathbf{1}$ & Parbhani & $16(40.00)$ & $12(30.00)$ & $12(30.00)$ \\
\hline $\mathbf{2}$ & Hingoli & $09(22.50)$ & $16(40.00)$ & $15(37.50)$ \\
\hline $\mathbf{3}$ & Nanded & $13(32.50)$ & $16(40.00)$ & $11(27.50)$ \\
\hline $\mathbf{4}$ & Beed & $16(40.00)$ & $15(37.50)$ & $09(22.50)$ \\
\hline $\mathbf{5}$ & Jalna & $10(25.00)$ & $22(55.00)$ & $08(20.00)$ \\
\hline $\mathbf{6}$ & Osmanabad & $14(35.00)$ & $14(35.00)$ & $12(30.00)$ \\
\hline $\mathbf{7}$ & Latur & $07(17.50)$ & $17(42.50)$ & $16(40.00)$ \\
\hline $\mathbf{8}$ & Aurangabad & $16(40.00)$ & $15(37.50)$ & $09(22.50)$ \\
\hline & $\begin{array}{l}\text { Marathwada } \\
\text { (N=320) }\end{array}$ & $\mathbf{1 0 1}(\mathbf{3 1 . 5 6})$ & $\mathbf{1 2 7}(\mathbf{3 9 . 6 8})$ & $\mathbf{9 2}(\mathbf{2 8 . 7 6})$ \\
\hline
\end{tabular}

* Figures in the parenthesis indicates the percentage 
Table.3 Distribution of the victims according to their educational level

\begin{tabular}{|c|c|c|c|c|c|c|c|c|}
\hline \multirow{2}{*}{$\begin{array}{l}\text { Sr. } \\
\text { No. }\end{array}$} & \multirow[t]{2}{*}{ Districts } & \multicolumn{7}{|c|}{ Education } \\
\hline & & Illiterate & $\begin{array}{c}\text { Primary } \\
\text { school }\end{array}$ & $\begin{array}{c}\text { Middle } \\
\text { school }\end{array}$ & $\begin{array}{l}\text { High } \\
\text { school }\end{array}$ & $\begin{array}{l}\text { Higher } \\
\text { school }\end{array}$ & $\begin{array}{l}\text { Gradu } \\
\text { ation }\end{array}$ & $\begin{array}{c}\text { Post } \\
\text { Graduation }\end{array}$ \\
\hline 1 & Parbhani & $\begin{array}{c}12 \\
(30.00)\end{array}$ & $\begin{array}{c}11 \\
(27.50)\end{array}$ & $\begin{array}{c}06 \\
(15.00)\end{array}$ & $\begin{array}{c}06 \\
(15.00)\end{array}$ & $\begin{array}{c}04 \\
(10.00)\end{array}$ & $\begin{array}{c}01 \\
(2.50)\end{array}$ & - \\
\hline 2 & Hingoli & $\begin{array}{c}15 \\
(37.50)\end{array}$ & $\begin{array}{c}10 \\
(25.00)\end{array}$ & $\begin{array}{c}02 \\
(5.00)\end{array}$ & $\begin{array}{c}06 \\
(15.00)\end{array}$ & $\begin{array}{c}04 \\
(10.00)\end{array}$ & $\begin{array}{c}03 \\
(7.50)\end{array}$ & - \\
\hline 3 & Nanded & $\begin{array}{c}06 \\
(15.00)\end{array}$ & $\begin{array}{c}11 \\
(27.50)\end{array}$ & $\begin{array}{c}02 \\
(5.00)\end{array}$ & $\begin{array}{c}11 \\
(27.50)\end{array}$ & $\begin{array}{c}07 \\
(17.50)\end{array}$ & $\begin{array}{c}03 \\
(7.50)\end{array}$ & - \\
\hline 4 & Beed & $\begin{array}{c}13 \\
(32.50)\end{array}$ & $\begin{array}{c}04 \\
(10.00)\end{array}$ & $\begin{array}{c}02 \\
(5.00)\end{array}$ & $\begin{array}{c}08 \\
(20.00)\end{array}$ & $\begin{array}{c}09 \\
(22.50)\end{array}$ & $\begin{array}{c}04 \\
(10.00)\end{array}$ & - \\
\hline 5 & Jalna & $\begin{array}{c}09 \\
(22.50)\end{array}$ & $\begin{array}{c}10 \\
(25.00)\end{array}$ & $\begin{array}{c}04 \\
(10.00)\end{array}$ & $\begin{array}{c}12 \\
(30.00)\end{array}$ & $\begin{array}{c}04 \\
(10.00)\end{array}$ & $\begin{array}{c}00 \\
(00.00)\end{array}$ & $\begin{array}{c}01 \\
(02.50)\end{array}$ \\
\hline 6 & Osmanabad & $\begin{array}{c}08 \\
(20.00)\end{array}$ & $\begin{array}{c}07 \\
(17.50)\end{array}$ & $\begin{array}{c}01 \\
(2.50)\end{array}$ & $\begin{array}{c}12 \\
(30.00)\end{array}$ & $\begin{array}{c}08 \\
(20.00)\end{array}$ & $\begin{array}{c}03 \\
(07.50)\end{array}$ & $\begin{array}{c}01 \\
(02.50)\end{array}$ \\
\hline 7 & Latur & - & $\begin{array}{c}07 \\
(17.50)\end{array}$ & $\begin{array}{c}10 \\
(25.00)\end{array}$ & $\begin{array}{c}09 \\
(22.50)\end{array}$ & $\begin{array}{c}12 \\
(30.00)\end{array}$ & $\begin{array}{c}02 \\
(05.00)\end{array}$ & - \\
\hline 8 & Aurangabad & $\begin{array}{c}03 \\
(07.50)\end{array}$ & $\begin{array}{c}08 \\
(20.00)\end{array}$ & $\begin{array}{c}12 \\
(30.00)\end{array}$ & $\begin{array}{c}06 \\
(15.00)\end{array}$ & $\begin{array}{c}10 \\
(25.00)\end{array}$ & $\begin{array}{c}01 \\
(02.50)\end{array}$ & - \\
\hline & $\begin{array}{l}\text { Marathwada } \\
(\mathbf{N}=320)\end{array}$ & $\begin{array}{c}66 \\
(20.62)\end{array}$ & $\begin{array}{c}68 \\
(21.25)\end{array}$ & $\begin{array}{c}39 \\
(12.18)\end{array}$ & $\begin{array}{c}70 \\
(21.87)\end{array}$ & $\begin{array}{c}58 \\
(18.13)\end{array}$ & $\begin{array}{c}17 \\
(5.32)\end{array}$ & $\begin{array}{c}\mathbf{0 2} \\
(\mathbf{0 . 6 3})\end{array}$ \\
\hline
\end{tabular}

* Figures in the parenthesis indicates the percentage

Table.4 Distribution of the victims according to their land holding

\begin{tabular}{|c|c|c|c|c|c|c|}
\hline \multirow{2}{*}{$\begin{array}{l}\text { Sr. } \\
\text { No }\end{array}$} & \multirow[t]{2}{*}{ District } & \multicolumn{5}{|c|}{ Land holding } \\
\hline & & $\begin{array}{l}\text { Marginal } \\
\text { (Up to1.00) }\end{array}$ & $\begin{array}{c}\text { Small } \\
(1.01 \text { to2.00) }\end{array}$ & $\begin{array}{c}\text { Semi- } \\
\text { medium } \\
(2.01 \text { to } 4.00)\end{array}$ & $\begin{array}{l}\text { Medium } \\
\text { (4.01 to10.0) }\end{array}$ & $\begin{array}{c}\text { Large } \\
\text { (Above 10.00) }\end{array}$ \\
\hline 1 & Parbhani & $15(37.50)$ & $16(40.00)$ & $07(17.50)$ & $02(05.00)$ & - \\
\hline 2 & Hingoli & $15(37.50)$ & $16(40.00)$ & $06(15.00)$ & $02(05.00)$ & $01(2.50)$ \\
\hline 3 & Nanded & $13(32.50)$ & $19(47.50)$ & $05(12.50)$ & $03(07.50)$ & - \\
\hline 4 & Beed & $15(37.50)$ & $12(30.00)$ & $09(22.50)$ & $04(10.00)$ & - \\
\hline 5 & Jalna & $14(35.00)$ & $15(37.50)$ & $05(12.50)$ & $04(10.00)$ & $02(5.00)$ \\
\hline 6 & Osmanabad & $13(32.50)$ & $14(35.00)$ & $10(25.00)$ & $03(07.50)$ & - \\
\hline 7 & Latur & $06(15.00)$ & $18(45.00)$ & $14(35.00)$ & $02(05.00)$ & - \\
\hline 8 & Aurangabad & $10(25.00)$ & $17(42.50)$ & $12(30.00)$ & $01(02.50)$ & - \\
\hline & $\begin{array}{l}\text { Marathwada } \\
(\mathrm{N}=\mathbf{3 2 0})\end{array}$ & $101(31.57)$ & $127(39.68)$ & $68(21.25)$ & $21(6.56)$ & $03(0.94)$ \\
\hline
\end{tabular}

* Figures in the parenthesis indicates the percentage 
Table.5 Distribution of the victims according to their subsidiary occupations

\begin{tabular}{|c|c|c|c|c|c|c|}
\hline \multirow{2}{*}{$\begin{array}{l}\text { Sr. } \\
\text { No }\end{array}$} & \multirow[t]{2}{*}{ Districts } & \multicolumn{5}{|c|}{ Subsidiary occupation } \\
\hline & & $\begin{array}{c}\text { Agriculture+ } \\
\text { Labour }\end{array}$ & $\begin{array}{c}\text { Agriculture } \\
\text { (farming) }\end{array}$ & $\begin{array}{c}\text { Agriculture }+ \text { allied } \\
\text { occupation }\end{array}$ & $\begin{array}{c}\text { Agriculture } \\
+ \text { Business }\end{array}$ & $\begin{array}{l}\text { Agriculture } \\
\text { + service }\end{array}$ \\
\hline 1 & Parbhani & $32(80.00)$ & $07(17.50)$ & - & - & $01(02.50)$ \\
\hline 2 & Hingoli & $26(65.00)$ & $09(22.50)$ & - & $05(12.50)$ & - \\
\hline 3 & Nanded & $24(60.00)$ & $05(12.50)$ & $01(02.50)$ & $06(15.00)$ & $04(10.00)$ \\
\hline 4 & Beed & $21(52.50)$ & $09(22.50)$ & $01(02.50)$ & $08(20.00)$ & $01(02.50)$ \\
\hline 5 & Jalna & $34(85.00)$ & $05(12.50)$ & - & $01(02.50)$ & - \\
\hline 6 & Osmanabad & $22(55.00)$ & $14(35.00)$ & - & $04(10.00)$ & - \\
\hline 7 & Latur & $17(42.50)$ & $08(20.00)$ & $09(22.50)$ & $04(10.00)$ & $02(05.00)$ \\
\hline 8 & Aurangabad & $20(50.00)$ & $13(32.50)$ & $02(05.00)$ & $05(12.50)$ & - \\
\hline & $\begin{array}{l}\text { Marathwada } \\
(\mathrm{N}=320)\end{array}$ & $196(61.25)$ & $70(21.88)$ & $13(04.06)$ & $33(10.31)$ & $08(02.50)$ \\
\hline
\end{tabular}

* Figures in the parenthesis indicates the percentage

Table.6 Distribution of the victims according to their annual income

\begin{tabular}{|c|c|c|c|c|c|c|c|}
\hline \multirow{2}{*}{$\begin{array}{l}\text { Sr. } \\
\text { No. }\end{array}$} & \multirow[t]{2}{*}{ District } & \multicolumn{6}{|c|}{ Annual Income (Rs.) } \\
\hline & & $\begin{array}{c}\text { Rs. } 27,000 /- \\
\text { (i.e. BPL) }\end{array}$ & $\begin{array}{l}27,001 /- \text { to } \\
50,000 /-\end{array}$ & $\begin{array}{c}50,001 /- \\
\text { to } \\
1,50,000 /-\end{array}$ & $\begin{array}{c}1,50,001 /- \\
\text { to } \\
2,50,000 /-\end{array}$ & $\begin{array}{c}2,50,001 /- \\
\text { to } \\
6,00,000 /-\end{array}$ & $\begin{array}{c}\text { Above } \\
6,00,000 /- \\
\text { (i.e. above } \\
\text { EBC) }\end{array}$ \\
\hline 1 & Parbhani & $\begin{array}{c}03 \\
(07.50)\end{array}$ & $\begin{array}{c}06 \\
(15.00)\end{array}$ & $\begin{array}{c}20 \\
(50.00)\end{array}$ & $\begin{array}{c}08 \\
(20.00)\end{array}$ & $\begin{array}{c}03 \\
(07.50)\end{array}$ & - \\
\hline 2 & Hingoli & $\begin{array}{c}02 \\
(05.50)\end{array}$ & $\begin{array}{c}11 \\
(27.50)\end{array}$ & $\begin{array}{c}17 \\
(42.50)\end{array}$ & $\begin{array}{c}06 \\
(15.00)\end{array}$ & $\begin{array}{c}04 \\
(10.00)\end{array}$ & - \\
\hline 3 & Nanded & $\begin{array}{c}01 \\
(02.50)\end{array}$ & $\begin{array}{c}12 \\
(30.00)\end{array}$ & $\begin{array}{c}21 \\
(52.50)\end{array}$ & $\begin{array}{c}04 \\
(10.00)\end{array}$ & $\begin{array}{c}02 \\
(05.00)\end{array}$ & - \\
\hline 4 & Beed & $\begin{array}{c}02 \\
(05.00)\end{array}$ & $\begin{array}{c}04 \\
(10.00)\end{array}$ & $\begin{array}{c}14 \\
(35.00)\end{array}$ & $\begin{array}{c}10 \\
(25.00)\end{array}$ & $\begin{array}{c}07 \\
(17.50)\end{array}$ & $\begin{array}{c}03 \\
(07.50)\end{array}$ \\
\hline 5 & Jalna & $\begin{array}{c}06 \\
(15.00)\end{array}$ & $\begin{array}{c}09 \\
(22.50)\end{array}$ & $\begin{array}{c}21 \\
(52.50)\end{array}$ & $\begin{array}{c}03 \\
(07.50)\end{array}$ & $\begin{array}{c}01 \\
(02.50)\end{array}$ & - \\
\hline 6 & Osmanabad & $\begin{array}{c}04 \\
(10.00)\end{array}$ & $\begin{array}{c}08 \\
(20.00)\end{array}$ & $\begin{array}{c}23 \\
(57.50)\end{array}$ & $\begin{array}{c}02 \\
(05.00)\end{array}$ & $\begin{array}{c}02 \\
(05.00)\end{array}$ & $\begin{array}{c}01 \\
(02.50)\end{array}$ \\
\hline 7 & Latur & $\begin{array}{c}01 \\
(02.50)\end{array}$ & $\begin{array}{c}11 \\
(27.50)\end{array}$ & $\begin{array}{c}26 \\
(72.50)\end{array}$ & - & - & - \\
\hline 8 & Aurangabad & $\begin{array}{c}00 \\
(00.00)\end{array}$ & $\begin{array}{c}09 \\
(22.50)\end{array}$ & $\begin{array}{c}15 \\
(37.50)\end{array}$ & $\begin{array}{c}11 \\
(27.50)\end{array}$ & $\begin{array}{c}05 \\
(12.50)\end{array}$ & - \\
\hline & $\begin{array}{l}\text { Marathwada } \\
(\mathrm{N}=320)\end{array}$ & $\begin{array}{c}19 \\
(5.94)\end{array}$ & $\begin{array}{c}70 \\
(21.87)\end{array}$ & $\begin{array}{c}157 \\
(49.07)\end{array}$ & $\begin{array}{c}46 \\
(14.37)\end{array}$ & $\begin{array}{c}24 \\
(7.50)\end{array}$ & $\begin{array}{c}04 \\
(01.25)\end{array}$ \\
\hline
\end{tabular}

* Figures in the parenthesis indicates the percentage 
Table.7 Distribution of the victims according to their irrigation facility

\begin{tabular}{|c|c|c|c|c|c|c|c|c|}
\hline \multirow{2}{*}{$\begin{array}{l}\text { Sr. } \\
\text { No }\end{array}$} & \multirow[t]{2}{*}{ District } & \multicolumn{7}{|c|}{ Irrigation facilities } \\
\hline & & No source & $\begin{array}{c}\text { Dam/K.T } \\
\text { Embankment }\end{array}$ & Canal & Farm Pond & $\begin{array}{l}\text { Well/Tube } \\
\text { Well }\end{array}$ & Lake & River \\
\hline 1 & Parbhani & $\begin{array}{c}19 \\
(47.50)\end{array}$ & - & $\begin{array}{c}06 \\
(15.00)\end{array}$ & - & $\begin{array}{c}11 \\
(27.50)\end{array}$ & - & $\begin{array}{c}04 \\
(10.00)\end{array}$ \\
\hline 2 & Hingoli & $\begin{array}{c}19 \\
(47.50)\end{array}$ & - & $\begin{array}{c}06 \\
(15.00)\end{array}$ & - & $\begin{array}{c}14 \\
(35.00)\end{array}$ & - & $\begin{array}{c}01 \\
(2.50)\end{array}$ \\
\hline 3 & Nanded & $\begin{array}{c}32 \\
(80.00)\end{array}$ & - & $\begin{array}{c}01 \\
(2.50)\end{array}$ & - & $\begin{array}{c}04 \\
(10.00)\end{array}$ & - & $\begin{array}{c}03 \\
(7.50)\end{array}$ \\
\hline 4 & Beed & $\begin{array}{c}16 \\
(40.00)\end{array}$ & $\begin{array}{c}02 \\
(5.00)\end{array}$ & $\begin{array}{c}04 \\
(10.00)\end{array}$ & - & $\begin{array}{c}16 \\
(40.00)\end{array}$ & $\begin{array}{c}01 \\
(2.50)\end{array}$ & $\begin{array}{c}01 \\
(2.50)\end{array}$ \\
\hline 5 & Jalna & $\begin{array}{c}34 \\
(85.00)\end{array}$ & $\begin{array}{c}01 \\
(2.50)\end{array}$ & $\begin{array}{c}04 \\
(10.00)\end{array}$ & $\begin{array}{c}01 \\
(2.50)\end{array}$ & - & - & - \\
\hline 6 & Osmanabad & $\begin{array}{c}24 \\
(60.00)\end{array}$ & $\begin{array}{c}01 \\
(2.50)\end{array}$ & - & - & $\begin{array}{c}15 \\
(37.50)\end{array}$ & - & - \\
\hline 7 & Latur & $\begin{array}{c}11 \\
(27.50)\end{array}$ & - & $\begin{array}{c}02 \\
(5.00)\end{array}$ & - & $\begin{array}{c}27 \\
(67.50)\end{array}$ & - & - \\
\hline 8 & Aurangabad & $\begin{array}{c}17 \\
(42.50)\end{array}$ & - & $\begin{array}{c}09 \\
(22.50)\end{array}$ & - & $\begin{array}{c}14 \\
(35.00)\end{array}$ & - & - \\
\hline & $\begin{array}{l}\text { Marathwada } \\
(\mathrm{N}=320)\end{array}$ & $\begin{array}{c}172 \\
(53.74)\end{array}$ & $\begin{array}{c}04 \\
(1.25)\end{array}$ & $\begin{array}{c}32 \\
(10.00)\end{array}$ & $\begin{array}{c}01 \\
(0.32)\end{array}$ & $\begin{array}{c}101 \\
(31.56)\end{array}$ & $\begin{array}{c}01 \\
(0.32)\end{array}$ & $\begin{array}{c}09 \\
(2.81)\end{array}$ \\
\hline
\end{tabular}

* Figures in the parenthesis indicates the percentage

Table.8 Types of cropping system adopted by victims farmers (Overall Marathwada)

\begin{tabular}{|c|l|c|c|}
\hline $\begin{array}{c}\text { Sr. } \\
\text { No. }\end{array}$ & Types cropping system & No. of victims & $\begin{array}{c}\text { \% of the } \\
\text { victims }(\mathbf{N}=320)\end{array}$ \\
\hline $\mathbf{1}$ & Sole cropping & 263 & 82.18 \\
\hline $\mathbf{2}$ & Intercropping & 57 & 17.82 \\
\hline
\end{tabular}

Table.9 Average productivity of the victims farmers (Overall Marathwada)

\begin{tabular}{|c|l|c|c|}
\hline $\begin{array}{c}\text { Sr. } \\
\text { No. }\end{array}$ & \multicolumn{1}{|c|}{ Season } & No. of victims & $\begin{array}{c}\text { Average productivity of } \\
\text { victims farmers (Qt/ha) }\end{array}$ \\
\hline A & Kharif & & \\
\hline $\mathbf{1}$ & Soybean & $198(61.78)$ & 9.56 \\
\hline $\mathbf{2}$ & Cotton & $183(57.18)$ & 11.20 \\
\hline $\mathbf{3}$ & Pigeonpea & $107(33.43)$ & 7.34 \\
\hline B & Rabi & & \\
\hline $\mathbf{1}$ & Bengal gram & $90(28.12)$ & 7.20 \\
\hline $\mathbf{2}$ & R. Sorghum & $99(30.93)$ & 9.65 \\
\hline $\mathbf{3}$ & Wheat & $52(16.25)$ & 11.99 \\
\hline
\end{tabular}


Table.10 Distribution of the victims according to their credit sources and indebtedness

(Marathwada, $\mathrm{N}=320$ )

\begin{tabular}{|c|c|c|c|c|c|}
\hline \multirow{2}{*}{$\begin{array}{l}\text { Sr. } \\
\text { No. }\end{array}$} & \multirow[t]{2}{*}{ Source } & \multicolumn{4}{|c|}{ Over all Marathwada region } \\
\hline & & $\begin{array}{l}\text { No of victim } \\
\text { taken credit }\end{array}$ & $\begin{array}{c}\text { Amount } \\
\text { in rupees } \\
\text { per } \\
\text { borrowing }\end{array}$ & $\begin{array}{l}\text { Outstanding } \\
\text { amount per } \\
\text { victim }\end{array}$ & $\begin{array}{c}\% \text { of } \\
\text { outstanding to } \\
\text { total credit } \\
\text { availed }\end{array}$ \\
\hline 1. & \multicolumn{5}{|l|}{ Institutional } \\
\hline a) & Co-operative bank & $85(26.56 \%)$ & 64560 & 84804 & 131 \\
\hline b) & Nationalize & $173(54.06 \%)$ & 120132 & 149032 & 124 \\
\hline c) & Rural bank & $37(11.56 \%)$ & 89167 & 112057 & 126 \\
\hline d) & Self-help group & $26(08.12 \%)$ & 45934 & 68451 & 149 \\
\hline 2) & \multicolumn{5}{|l|}{ Non-institutional } \\
\hline a) & $\begin{array}{l}\text { Private } \\
\text { moneylenders }\end{array}$ & $116(36.25 \%)$ & 221703 & 324031 & 146 \\
\hline b) & Friends and relatives & $91(28.43 \%)$ & 221814 & 270181 & 125 \\
\hline
\end{tabular}

Table.11 Distribution of the victims according to overall livelihood pattern

(Marathwada, $\mathrm{N}=320$ )

\begin{tabular}{|c|c|c|c|}
\hline \multirow[t]{2}{*}{ Sr. No. } & \multirow[t]{2}{*}{ Source } & \multicolumn{2}{|c|}{ Overall Marathwada Region } \\
\hline & & $\begin{array}{l}\text { No. of victims } \\
\text { families }\end{array}$ & Rs. per house hold \\
\hline & Income pattern & & \\
\hline 1 & Agriculture & $332(100.00 \%)$ & $74576 /-$ \\
\hline 2 & Allied occupation & $13(4.06 \%)$ & $21176 /-$ \\
\hline 3 & Wages earning & $196(61.25 \%)$ & $30988 /-$ \\
\hline \multirow[t]{3}{*}{4} & Non profession business & $41(12.81 \%)$ & $38080 /-$ \\
\hline & Total income (A) & & $152321 /-$ \\
\hline & Expenditure pattern & & \\
\hline 1 & Expense incurred on food & & 26812 /- \\
\hline \multirow[t]{3}{*}{2} & Expense incurred on total non-food & & $117353 /-$ \\
\hline & Total expenditure (B) & & $144165 /-$ \\
\hline & Surplus/deficit (+/-) (A-B) & & $8156 /-$ \\
\hline
\end{tabular}


Table.12 Distribution of victims according to their farming related causes of suicides

\begin{tabular}{|c|c|c|c|c|c|c|c|c|c|c|}
\hline $\begin{array}{l}\text { Sr. } \\
\text { No }\end{array}$ & $\begin{array}{l}\text { Farming related } \\
\text { causes }\end{array}$ & $\begin{array}{c}\text { PBN } \\
(\mathrm{N}=40)\end{array}$ & $\begin{array}{c}\text { HGL } \\
(\mathrm{N}=40)\end{array}$ & $\begin{array}{c}\text { NND } \\
(\mathrm{N}=\mathbf{4 0})\end{array}$ & $\begin{array}{l}\text { BEED } \\
(\mathrm{N}=40)\end{array}$ & $\begin{array}{c}\mathbf{J L N} \\
(\mathbf{N}=\mathbf{4 0})\end{array}$ & $\begin{array}{l}\mathrm{O}^{\prime} \mathrm{BAD} \\
(\mathrm{N}=40)\end{array}$ & $\begin{array}{c}\text { LTR } \\
(\mathrm{N}=\mathbf{4 0})\end{array}$ & $\begin{array}{l}\mathbf{A}^{\prime} \mathbf{B A D} \\
(\mathbf{N}=\mathbf{4 0})\end{array}$ & $\begin{array}{l}\text { Marathwada } \\
(\mathbf{N}=\mathbf{3 2 0})\end{array}$ \\
\hline 1 & $\begin{array}{l}\text { Crop failure due } \\
\text { to drought / lack } \\
\text { of irrigation }\end{array}$ & $\begin{array}{c}34 \\
(85.00)\end{array}$ & $\begin{array}{c}38 \\
(95.00)\end{array}$ & $\begin{array}{c}38 \\
(95.00)\end{array}$ & $\begin{array}{c}37 \\
(92.50)\end{array}$ & $\begin{array}{c}40 \\
(100.00)\end{array}$ & $\begin{array}{c}39 \\
(97.50)\end{array}$ & $\begin{array}{c}23 \\
(57.50)\end{array}$ & $\begin{array}{c}30 \\
(75.00)\end{array}$ & $\begin{array}{c}279 \\
(87.18)\end{array}$ \\
\hline 2 & $\begin{array}{l}\text { Crop failure due } \\
\text { to hailstorm }\end{array}$ & - & $\begin{array}{c}01 \\
(2.50)\end{array}$ & - & - & $\begin{array}{c}10 \\
(25.00)\end{array}$ & - & $\begin{array}{c}06 \\
(15.00)\end{array}$ & $\begin{array}{c}01 \\
(2.50)\end{array}$ & $\begin{array}{c}18 \\
(5.62)\end{array}$ \\
\hline 3 & $\begin{array}{l}\text { Crop failure due } \\
\text { to insect / disease } \\
\text { infestation }\end{array}$ & - & $\begin{array}{c}01 \\
(2.50)\end{array}$ & - & $\begin{array}{c}02 \\
(5.00)\end{array}$ & - & - & - & - & $\begin{array}{c}03 \\
(\mathbf{0 . 9 3})\end{array}$ \\
\hline 4 & $\begin{array}{l}\text { Crop failure due } \\
\text { to poor seed } \\
\text { germination }\end{array}$ & - & - & - & - & - & - & - & $\begin{array}{c}02 \\
(2.50)\end{array}$ & $\begin{array}{c}02 \\
(0.62)\end{array}$ \\
\hline 5 & $\begin{array}{l}\text { Crop failure due } \\
\text { to unseasonal } \\
\text { rains }\end{array}$ & - & - & - & - & - & - & $\begin{array}{c}09 \\
(22.05)\end{array}$ & - & $\begin{array}{c}09 \\
(2.81)\end{array}$ \\
\hline 6 & $\begin{array}{l}\text { Failure of open } \\
\text { well or tube well }\end{array}$ & - & - & - & $\begin{array}{c}05 \\
(12.50)\end{array}$ & - & - & $\begin{array}{c}02 \\
(5.00)\end{array}$ & $\begin{array}{c}03 \\
(7.50)\end{array}$ & $\begin{array}{c}10 \\
(3.12)\end{array}$ \\
\hline
\end{tabular}

Table.13 Distribution of victims according to their indebtedness related causes of suicides

\begin{tabular}{|c|c|c|c|c|c|c|c|c|c|c|}
\hline $\begin{array}{l}\text { Sr. } \\
\text { No. }\end{array}$ & $\begin{array}{c}\text { Indebtedness related } \\
\text { causes }\end{array}$ & $\begin{array}{c}\text { PNB } \\
(\mathrm{N}=40)\end{array}$ & $\begin{array}{c}\text { HGL } \\
(\mathbf{N}=40)\end{array}$ & $\begin{array}{l}\text { NND } \\
(\mathrm{N}=40)\end{array}$ & $\begin{array}{l}\text { BEED } \\
(\mathrm{N}=40)\end{array}$ & $\begin{array}{c}\text { JLN } \\
(\mathbf{N}=40)\end{array}$ & $\begin{array}{c}\mathrm{O}^{\prime} \mathrm{BA} \\
\mathrm{D} \\
(\mathrm{N}=40)\end{array}$ & $\begin{array}{c}\text { LTR } \\
(\mathbf{N}=40)\end{array}$ & $\begin{array}{l}\text { A'BAD }^{\prime} \text { BA } \\
(\mathrm{N}=40)\end{array}$ & $\begin{array}{c}\text { Marathwada } \\
(\mathbf{N}=320)\end{array}$ \\
\hline 1 & $\begin{array}{l}\text { Increased } \\
\text { indebtedness }\end{array}$ & $\begin{array}{c}32 \\
(80.00)\end{array}$ & $\begin{array}{c}29 \\
(72.50)\end{array}$ & $\begin{array}{c}34 \\
(85.00)\end{array}$ & $\begin{array}{c}37 \\
(92.50)\end{array}$ & $\begin{array}{c}40 \\
(100)\end{array}$ & $\begin{array}{c}32 \\
(80.00)\end{array}$ & $\begin{array}{c}01 \\
(2.50)\end{array}$ & $\begin{array}{c}40 \\
(100)\end{array}$ & $\begin{array}{c}245 \\
(76.56)\end{array}$ \\
\hline 2 & $\begin{array}{l}\text { Torturing by money } \\
\text { lender for } \\
\text { repayment of loan }\end{array}$ & $\begin{array}{c}03 \\
(7.50)\end{array}$ & $\begin{array}{c}04 \\
(10.00)\end{array}$ & $\begin{array}{c}15 \\
(37.50)\end{array}$ & $\begin{array}{c}14 \\
(35.00)\end{array}$ & $\begin{array}{c}10 \\
(25.00)\end{array}$ & $\begin{array}{c}04 \\
(10.00)\end{array}$ & $\begin{array}{c}01 \\
(2.50)\end{array}$ & $\begin{array}{c}18 \\
(45.00)\end{array}$ & $\begin{array}{c}69 \\
(21.56)\end{array}$ \\
\hline 3 & $\begin{array}{l}\text { Recovery pressure } \\
\text { from money lender }\end{array}$ & $\begin{array}{c}04 \\
(10.00)\end{array}$ & $\begin{array}{c}05 \\
(12.50) \\
\end{array}$ & $\begin{array}{c}09 \\
(22.50) \\
\end{array}$ & $\begin{array}{c}10 \\
(25.00)\end{array}$ & $\begin{array}{c}19 \\
(47.50)\end{array}$ & $\begin{array}{c}16 \\
(40.00)\end{array}$ & - & $\begin{array}{c}19 \\
(47.50)\end{array}$ & $\begin{array}{c}82 \\
(25.62)\end{array}$ \\
\hline 4 & $\begin{array}{l}\text { Humiliation at } \\
\text { public place by } \\
\text { money lender }\end{array}$ & $\begin{array}{c}04 \\
(10.00)\end{array}$ & $\begin{array}{c}02 \\
(2.50)\end{array}$ & $\begin{array}{c}06 \\
(15.00)\end{array}$ & $\begin{array}{c}05 \\
(12.50)\end{array}$ & $\begin{array}{c}05 \\
(12.50)\end{array}$ & $\begin{array}{c}02 \\
(5.00)\end{array}$ & $\begin{array}{c}02 \\
(2.50)\end{array}$ & $\begin{array}{c}03 \\
(7.50)\end{array}$ & $\begin{array}{c}29 \\
(09.06)\end{array}$ \\
\hline 5 & $\begin{array}{l}\text { Auction of assets/ } \\
\text { livestock / other } \\
\text { household stocks }\end{array}$ & - & - & $\begin{array}{c}01 \\
(2.50)\end{array}$ & $\begin{array}{c}04 \\
(10.00)\end{array}$ & - & - & $\begin{array}{c}17 \\
(42.50)\end{array}$ & - & $\begin{array}{c}22 \\
(06.87)\end{array}$ \\
\hline 6 & $\begin{array}{l}\text { Availment of loan } \\
\text { from non- } \\
\text { institutional sources }\end{array}$ & $\begin{array}{c}07 \\
(17.50)\end{array}$ & $\begin{array}{c}10 \\
(25.00)\end{array}$ & - & - & - & - & - & $\begin{array}{c}06 \\
(15.00)\end{array}$ & $\begin{array}{c}23 \\
(07.18)\end{array}$ \\
\hline
\end{tabular}


Table.14 Distribution of victims according to social and other causes related to the suicides

\begin{tabular}{|c|c|c|c|c|c|c|c|c|c|c|}
\hline $\begin{array}{l}\text { Sr. } \\
\text { No }\end{array}$ & Social \& other causes & $\begin{array}{c}\text { PNB } \\
(\mathrm{N}=40)\end{array}$ & $\begin{array}{c}\text { HGL } \\
(\mathrm{N}=40)\end{array}$ & $\begin{array}{l}\text { NND } \\
(\mathrm{N}=40)\end{array}$ & $\begin{array}{l}\text { BEED } \\
(\mathbf{N}=40)\end{array}$ & $\begin{array}{c}\text { JLN } \\
(\mathbf{N}=\mathbf{4 0})\end{array}$ & $\begin{array}{l}\begin{array}{l}\text { O'BAD } \\
(\mathrm{N}=40)\end{array} \\
\end{array}$ & \begin{tabular}{|c|} 
LTR \\
$(\mathbf{N}=\mathbf{4 0})$
\end{tabular} & $\begin{array}{l}\begin{array}{l}A^{\prime} \mathbf{B A D} \\
(\mathbf{N}=40)\end{array}\end{array}$ & $\begin{array}{c}\text { Marathwada } \\
(\mathbf{N}=\mathbf{3 2 0})\end{array}$ \\
\hline 1 & Dowry system & - & - & - & $\begin{array}{c}02 \\
(5.00)\end{array}$ & - & - & - & $\begin{array}{c}04 \\
(10.00)\end{array}$ & $\begin{array}{c}06 \\
(1.87)\end{array}$ \\
\hline 2 & $\begin{array}{l}\text { Drop in socio- } \\
\text { economic status }\end{array}$ & $\begin{array}{c}18 \\
(45.00)\end{array}$ & $\begin{array}{c}18 \\
(45.00)\end{array}$ & $\begin{array}{c}12 \\
(30.00)\end{array}$ & $\begin{array}{c}18 \\
(45.00)\end{array}$ & $\begin{array}{c}16 \\
(40.00)\end{array}$ & $\begin{array}{c}27 \\
(67.50)\end{array}$ & $\begin{array}{c}37 \\
(92.50)\end{array}$ & $\begin{array}{c}24 \\
(60.00)\end{array}$ & $\begin{array}{c}170 \\
(53.12)\end{array}$ \\
\hline 3 & $\begin{array}{l}\text { Marriageable age of } \\
\text { daughters / sisters }\end{array}$ & $\begin{array}{c}10 \\
(25.00)\end{array}$ & $\begin{array}{c}13 \\
(32.50)\end{array}$ & $\begin{array}{c}15 \\
(37.50)\end{array}$ & $\begin{array}{c}09 \\
(22.50)\end{array}$ & $\begin{array}{c}22 \\
(55.00)\end{array}$ & $\begin{array}{c}01 \\
(2.50)\end{array}$ & - & $\begin{array}{c}27 \\
(67.50)\end{array}$ & $\begin{array}{c}97 \\
(30.31)\end{array}$ \\
\hline 4 & $\begin{array}{l}\text { Suicide of family } \\
\text { member or any peers }\end{array}$ & $\begin{array}{c}08 \\
(20.00)\end{array}$ & $\begin{array}{c}14 \\
(35.00)\end{array}$ & $\begin{array}{c}09 \\
(22.50)\end{array}$ & $\begin{array}{c}08 \\
(20.00)\end{array}$ & $\begin{array}{c}14 \\
(35.00)\end{array}$ & $\begin{array}{c}09 \\
(22.50)\end{array}$ & - & $\begin{array}{c}03 \\
(7.50)\end{array}$ & $\begin{array}{c}65 \\
(20.31)\end{array}$ \\
\hline 5 & $\begin{array}{l}\text { Dispute with the } \\
\text { family member / } \\
\text { neighbour /others }\end{array}$ & $\begin{array}{c}05 \\
(12.50)\end{array}$ & $\begin{array}{c}02 \\
(5.00)\end{array}$ & $\begin{array}{c}06 \\
(15.00)\end{array}$ & $\begin{array}{c}06 \\
(15.00)\end{array}$ & $\begin{array}{c}01 \\
(2.50)\end{array}$ & $\begin{array}{c}03 \\
(7.50)\end{array}$ & $\begin{array}{c}01 \\
(2.50)\end{array}$ & $\begin{array}{c}07 \\
(17.50)\end{array}$ & $\begin{array}{c}31 \\
(9.69)\end{array}$ \\
\hline 6 & $\begin{array}{l}\text { Death of the family } \\
\text { member prior to } \\
\text { suicide }\end{array}$ & - & - & $\begin{array}{c}01 \\
(2.50)\end{array}$ & $\begin{array}{c}01 \\
(2.50)\end{array}$ & $\begin{array}{c}03 \\
(7.50)\end{array}$ & - & $\begin{array}{c}05 \\
(12.50)\end{array}$ & - & $\begin{array}{c}10 \\
(3.12)\end{array}$ \\
\hline 7 & $\begin{array}{l}\text { Chronic health } \\
\text { problem of their own } \\
\text { or family members }\end{array}$ & $\begin{array}{c}20 \\
(50.00)\end{array}$ & $\begin{array}{c}20 \\
(50.00)\end{array}$ & $\begin{array}{c}11 \\
(27.50)\end{array}$ & $\begin{array}{c}15 \\
(37.50)\end{array}$ & $\begin{array}{c}12 \\
(30.00)\end{array}$ & $\begin{array}{c}16 \\
(40.00)\end{array}$ & $\begin{array}{c}04 \\
(10.00)\end{array}$ & $\begin{array}{c}10 \\
(25.00)\end{array}$ & $\begin{array}{c}108 \\
(33.75)\end{array}$ \\
\hline 8 & Addictions of victim & $\begin{array}{c}11 \\
(27.50)\end{array}$ & $\begin{array}{c}23 \\
(57.50)\end{array}$ & $\begin{array}{c}22 \\
(55.00)\end{array}$ & $\begin{array}{c}20 \\
(50.00)\end{array}$ & $\begin{array}{c}12 \\
(30.00)\end{array}$ & $\begin{array}{c}15 \\
(37.50)\end{array}$ & $\begin{array}{c}01 \\
(2.50)\end{array}$ & $\begin{array}{c}24 \\
(60.00)\end{array}$ & $\begin{array}{c}128 \\
(40.00)\end{array}$ \\
\hline
\end{tabular}


Table.15 Suggestions of the family members of the deceased farmers to prevent farmers' suicide

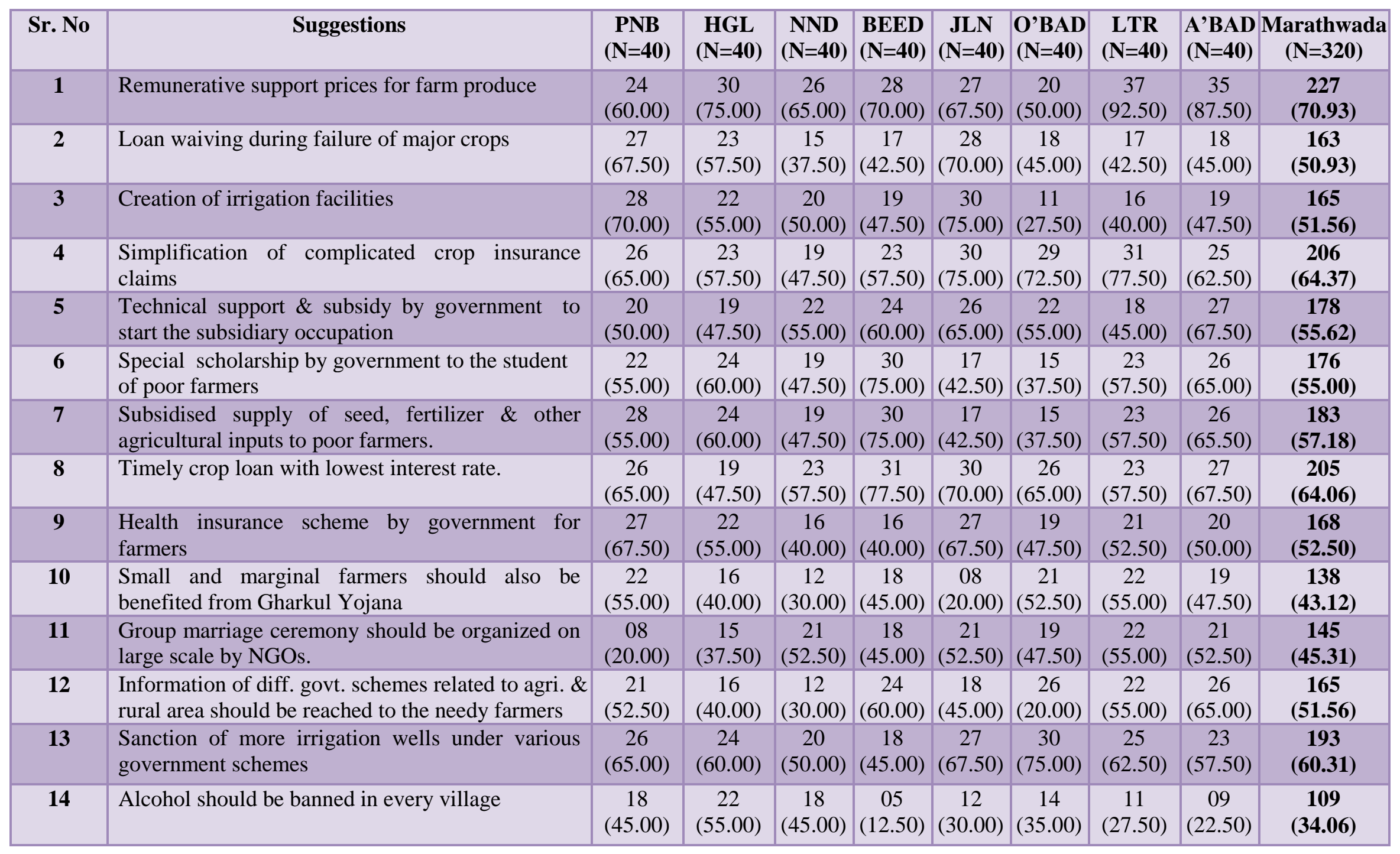




\section{Feedback from family members}

Table 15 depicts the suggestions given by victims' family members to prevent farmers' suicide. Data indicated that 70.93 per cent suggested for providing remunerative prices for farm produce, followed by simplification of crop insurance claims (64.37\%), timely crop loan with minimal interest rate (64.06\%), sanction of more irrigation wells under various government schemes (60.31\%), subsidised seed, fertilizer and other agricultural inputs to poor farmers $(57.18 \%)$, technical support and subsidy to start any subsidiary occupation (55.62\%), special educational scholarship by government to the students of poor farmers $(55.00 \%)$, health insurance scheme for farmers by the government $(52.50 \%)$, information of different government schemes related to agriculture and rural development should reached to the needy farmers $(51.56 \%)$, irrigation facilities should be created $(51.56 \%)$ and loan waiving during failure of major crops (50.93\%).

Similar results were obtained by Dandekar et al., (2005), Kale (2008) and Manjunatha and Ramappa (2017)

From a detailed study of farmer's suicides in Marathwada, it is concluded that crop failure due to drought / lack of irrigation was principal cause followed by high indebtedness.

Feedback from family members on how to prevent distress in the family leading to suicide revealed that provision of remunerative prices for farm produce, simplification of crop insurance claim, crop loans at lower interest rates, government support for digging wells and financial and technical support for taking up allied occupations emerged as the main suggestions.

\section{Implications}

Sole cropping is prominent in all the districts of Marathwada region with soyabean and cotton based cropping system under rainfed situation. On other hand very few of them were having subsidiary occupations. Therefore, it is implicated to initiate Integrated Farming System (IFS) which will diversify the income sources of marginal and small farmers.

Main cause of farmers' suicide was crop failure due to drought situation. Hence, it is recommended to create awareness and increase the participation of the farmers by various extension agencies to construct the farm ponds, recharge of wells / bores, water conservation techniques (Jal Ukat Shivar - a flagship programme of the Govt. of Maharashtra) and judicious use of available water through drip and sprinkler irrigation.

It is also recommended to provide psychological counselling facilities for severely distress farmers to change their mindset.

The present study revealed that social issues such as marriageable age of the daughters / childs also contribute farmers' distress. Therefore, it is recommended to create awareness about discontinuation of dowry system and organization of group marriages in rural areas through NGOs and social workers.

Skill training to poor farmers to take up subsidiary occupations.

\section{Acknowledgement}

We thank Indian Council of Agricultural Research - National Agricultural Science Fund (ICAR-NASF) project for providing the financial support for conducting the study. 


\section{References}

Dandekar, A., Narawada, S., Rathod, R., Ingle, R., Kulkarni, V. and Sateppa, V.D. 2005. Causes of Farmer Suicides in Maharashtra: An Enquiry. Final Report submitted to the Mumbai High Court, Tata Institute of Social Sciences, Tuljapur, Osamanabad.

Kale, N.M. 2008. Socio-economic, Psychological and Situational Causes of Suicides of Farmers in Vidarbha Region. Ph.D. (Thesis) submitted to Dr.
Panjabrao Deshmukh Krishi Vidyapeeth, Akola.

Manjunatha, A.V. and K.B. Ramappa 2017. Farmer Suicide: An All India Study. Agriculture Development and Rural Transformation Centre Report, Institute for Social and Economic Change, Karnataka.

National Crime Records Bureau 2017. Accidental Deaths and Suicides Reports of Various Years. Retrieved from http://ncrb.nic.in

\section{How to cite this article:}

Venkateswarlu, B., R.D. Ahire and Kapse, P.S. 2019. Farmers Suicides in Marathwada Region of India: A Causative Analysis. Int.J.Curr.Microbiol.App.Sci. 8(04): 296-308. doi: https://doi.org/10.20546/ijcmas.2019.804.034 